\title{
Design and Implementation of Power Plant Coal Transmission Control Information Linkage System Based on $\mathrm{VC}++$
}

\author{
Lei Sun ${ }^{1}$, Qingsu $\operatorname{Sun}^{2}$ \\ ${ }^{1}$ Jiangsu City Vocational College, 210036, Nanjing, Jiangsu, China \\ ${ }^{2}$ Nanjing Jillian Software Technology Corp, 210061, Nanjing, Jiangsu, China
}

\begin{abstract}
The implementation of coal linkage program control system and video monitoring system in power plant, which can improve the stable operation of coal handling system, In this paper, the design of a power plant coal handling control information linkage system, Introduces the structure, function and characteristics of the system and Discusses the key technology of receiving and sending, data analysis and display data in $\mathrm{VC}++$ environment, The practical application shows that the system is practical and reliable, good operation.
\end{abstract}

Keywords. VC++; Acquisition; Coal Transmission Control; linkage System

\section{Introduction}

Coal Transmission System has an important role in the power plant.Without reliable coal supply, it is impossible to keep the operation of steam turbine, boiler, electricity from failure. Based on the features of the coal transmission, it is critical to ensure the equipment operating smoothly. Without a wonderful circumstance of the equipment, it is meaningless to talk about the safety and security. In general the structure features of transmission system equipment are: long belts, long lines, many sets and complicated transmission ways. Now most of power plants will supervise the belt, magnetic separator, coal crusher and some else equipment by PLC monitor and video monitor, so as to accomplish the alarming protection of automatic coal supply, coal blending and coal batching, even the belt offset, tightening, speeding, slipping,deviation. However, the disadvantage of control system is that the field controller can only see the feedback from the field equipment without noting the practical operation circumstance of the equipment. Therefore most of coal transmission system will install the video monitor system and the industrial television monitor system, so that they can see the situation of the field coal transmission, which improves the operation situation and the stability of the equipment. Meanwhile it will considerably reduce the time of the operator inspecting the field, and ensure the safety and stability of the sets in the transmission process. Through investigating the above, In the most of power plants, their transmission control system and the video monitor system are separated system. For this, the operator in the control room must control them separately and shift each other to keep a better monitor. Then it increases the working strength of the operators, and can't ensure the safety and stability of the coal transmission equipment. After we combine them as a single unit, the stability will be improved. Once the equipment is unstable or the controller is operating an important sets, the monitor system can turn 
its head to the sets and show the situation on the screen of TV, so that they can monitor the situation of the sets, reducing the mistake rates and the worker's working strength.

\section{The system structure and function design}

\subsection{The main function of the software system}

Coal transmission control information linkage system combined the video monitor system and the coal transmission control system for a linkage control with each other, which means that can be monitored when the operator goes on his work as the order of coal supply, stop coal or something wrong occurs in the important sets which shown in the screen of the monitor. In the whole process, the operator only needs to operate the linkage system instead of operating the relative video monitors to finish the automatic shift and monitor of the field picture. In this way, the monitor ensures its real-time and visual advantage, decreasing the complex operating steps and without the expanding set failure caused by the unreal-time monitor, which extremely improve the working efficiency of operators.

(1)Data receive $\mathrm{e}^{[2]}$. It is the first step to implement the software function, starting the multiplethreading, monitoring the front end data acquisition and upload automatically, monitoring the linkage requirement from the client, then complete the receive and dealing by creating the message-responding function.

(2) Data analyze. To make sure the data accurate, real and reliable, it will send the failure data by MOBUS protocol format. And it must be analyzed by the requirement of protocol format as the software is accepting the data.

(3) Data vision. Display the received data on the big screen.

(4) Data store. Store the data as text, and using the derived classes of class VC++ CFile to store the files in the system.

(5) Historical data. This system adopts the derived classes of class VC++ CFile to display all historical data.

\subsection{System construction drawing}

The software system is divided to acquisition module and control module. The acquisition module can display a single view and multiple view modules to be browsed in a real-time way. Besides the control part will make the acquisition date be produced as pictures to be viewed. The system adopts $\mathrm{C} / \mathrm{S}$ structure, the front end uses 232/485 communication, and the linkage picture uses TCP/IP. The software system construction drawing is fig 1 .

\section{Critical technology}

\subsection{Receiving and sending of data}

It adopts $\mathrm{VC}++$ based on the communication unit of Socket internet, and the corresponding communication construction will be built efficiently according to the concrete situation of the power plants $^{[4]}$. The progress is shown as fig 2 and fig 3 .

Like fig 3, m_bConnected indicates weather the internet is connected successfully, m_sBuffer[4096] Indicates the variable values of sending data buffer, m_sRevBuffer[4096] indicates the variable values of receiving data buffer; OnConnect()indicates Socket contiguous function, OnClose()Socket indicates close function, OnReceive()indicates data receiving function, Onsend()indicates data sending function. 


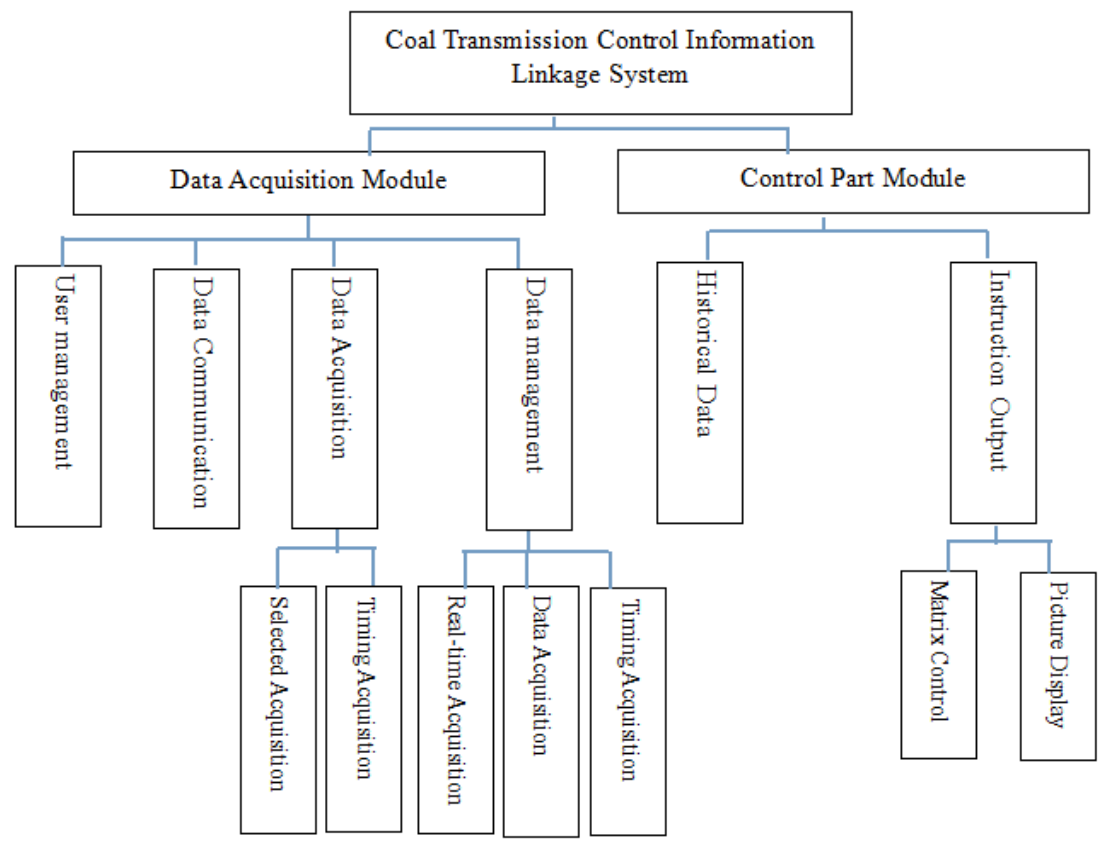

Figure 1. Software construction drawing

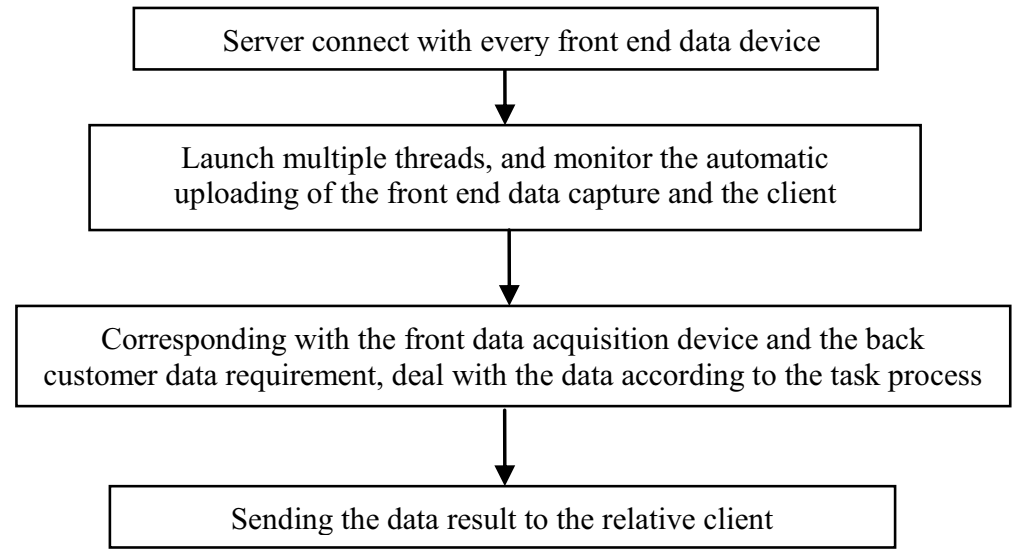

Figure 2. Data receiving and sending process

\begin{tabular}{|l|}
\hline With PLC communication and Socket Control Unit Class \\
\hline +m_bConnected : bool \\
+m_sBuffer [4096] : char \\
+m_sRecBuffer [4096] : char \\
\hline +OnConnect () \\
+OnReceive () \\
+OnSend () \\
+OnClose ()
\end{tabular}

Figure 3. With PLC communication and Socket control unit class 


\subsection{The intelligent interaction between failure point and photograph point}

Adopting the independent research tablet protocol, building the model based on XML file tree, every failure point and photograph point can have an efficient intelligent interaction. The XML file shown their interaction is as follow:

$<$ ?xml version= "1.0" encoding= "gb2312"? $>$

$<$ biaoqian $>$

$<$ title $>$ guanlian $</$ title $>$

$<$ Device id= "1" name= "belt 9 first head" $>$

$</$ Device $>$

$</$ biaoqian $>$

\subsection{Data analysis}

Adopting this process to analyze about the failure point information can make sure the new failure point picture can be always shown on the screen.

if (new failure point) then

\{ shift the pictures from the top left corner, put the original picture to the backward. The first one moves to the second, the second moves to the third, and so on $\}$

\section{System interface design}

Coal Transmission Control Information Linkage System is as fig 4. The linkage system action a failure pictures will be shown at the big screen when the failure happened. As fig 4 and 5:

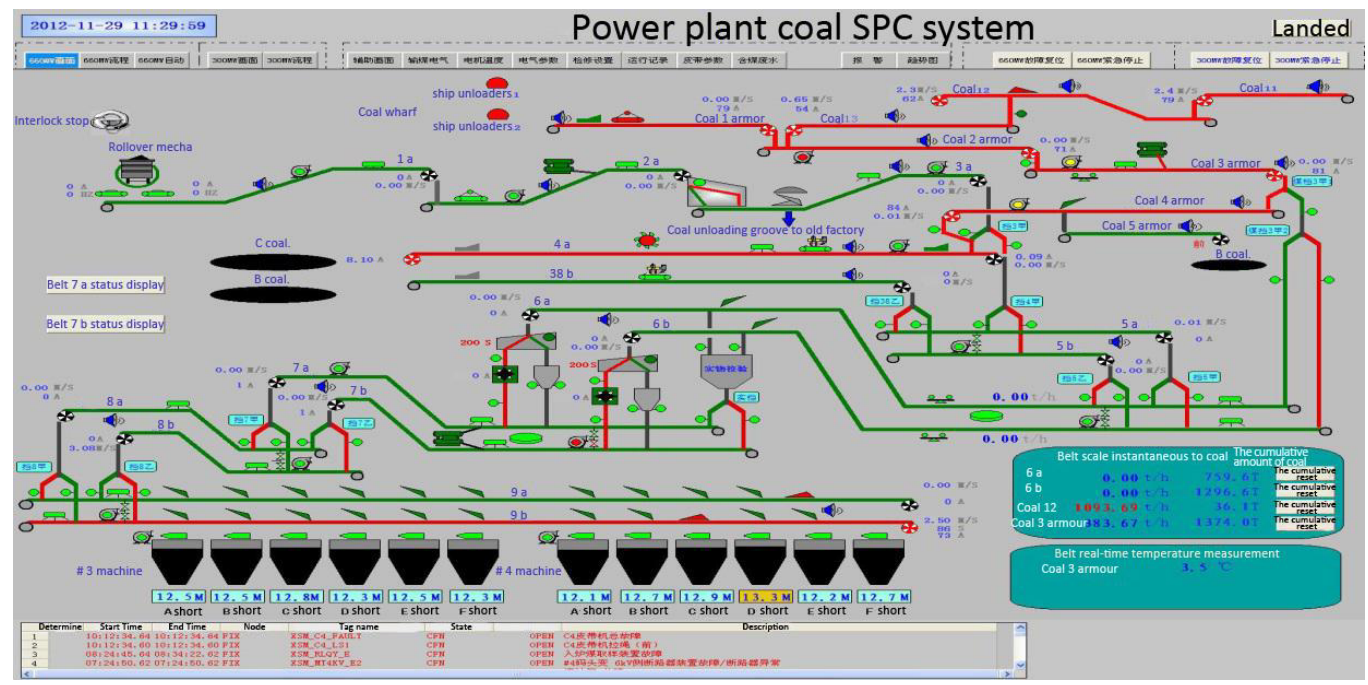

Figure 4. Power plant coal transmission control information linkage system 


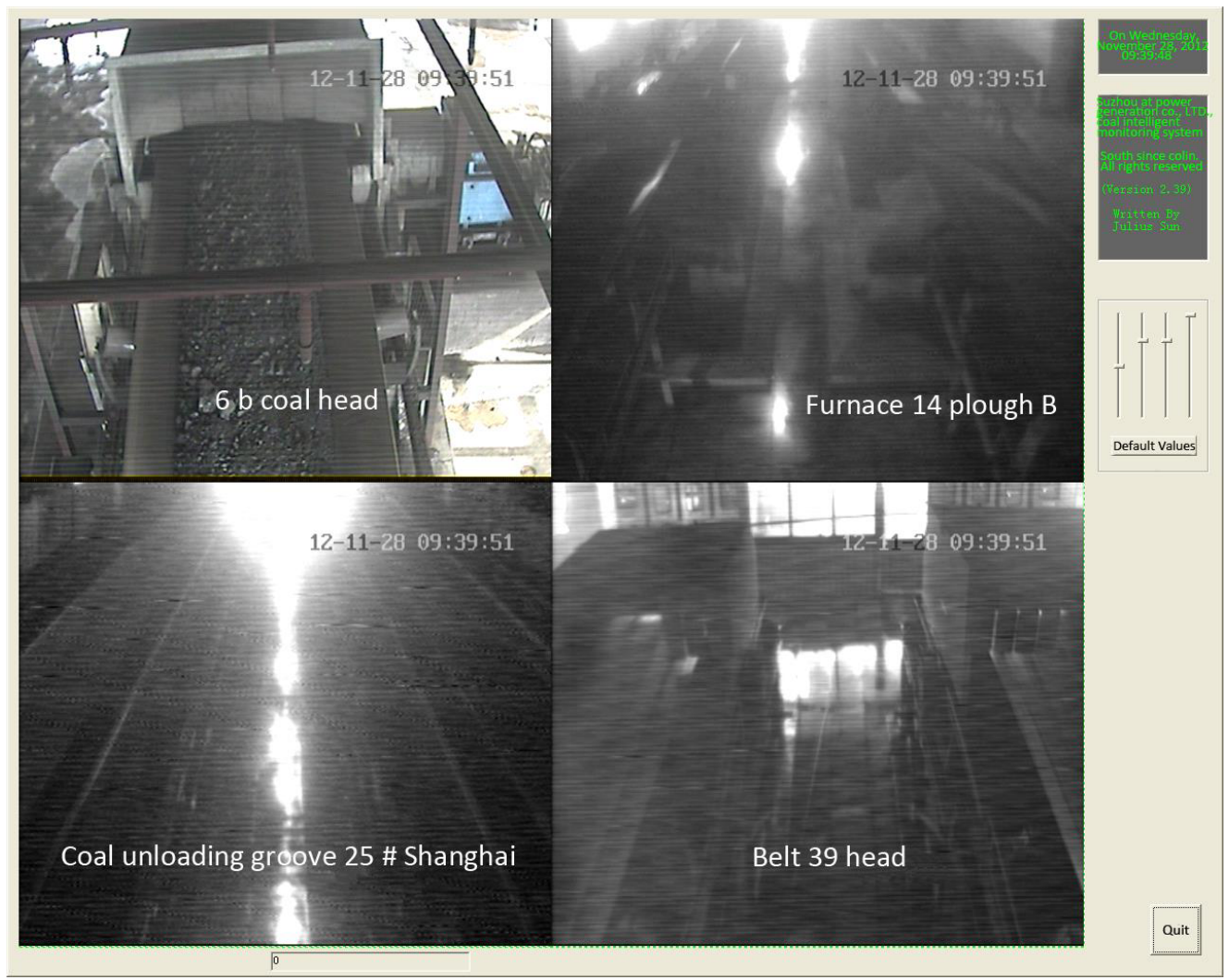

Figure 5. The failure picture on the monitor

\section{Conclusion}

Based on $\mathrm{VC}++$ Power plant coal transmission control information linkage system, the captured failure point will be sent to the video control system by socket, and shown at the interface of the monitor, so that the video monitor and power plant coal transmission control information linkage system can be combined, and they can interact with each other. The system can be benefit to the operator to find the failure of devices or keep a inspecting view as the important device produced actions, so that the accidents can be lessened. It ensures the real-time, visual feature, and reduces the complicated operating process for the operator, avoiding the failure expanding its effects, improving the workers' working efficiency.

\section{References}

1. Cheng Deqiang, He Yuanqing, An Duo, Niu Hezhu.The Linkage of Coal Mine Automation and Intelligent Video Surveillance System [J]. Safety in Coal Mine, 2013, 43(6):73-75.

2. Zhang Kehan, Zhang Huhe, Gu Lifeng. Motor Condition Monitoring Software Design Under $\mathrm{VC}++[\mathrm{J}]$. Measure and Control Technique, 2012(2): 23-27.

3. Chai Gaiping, Li Longmao, Jiang Zhihong. Ore Granularity Detection System Design Based on Image Processing [J]. Metallurgic Industry Automation, 2013, 37(6): 63-66.

4. Li Ming. Coal-mining Machine Intelligent Supervisor System Design Based on $\mathrm{VC}++[\mathrm{J}]$. Coal Mining Machinery, 2013, 34(9): 245-247. 